\title{
HISTORIOGRAFÍA DE LA ENFERMERÍA EN ESPAÑ̃A: LA HISTORIA DE UNA PEQUEÑA-GRAN FAMILIA
}

\author{
Francisca J. Hernández Martín \\ Profesora Titular de la Escuela de Enfermería. \\ Universidad Complutense de Madrid
}

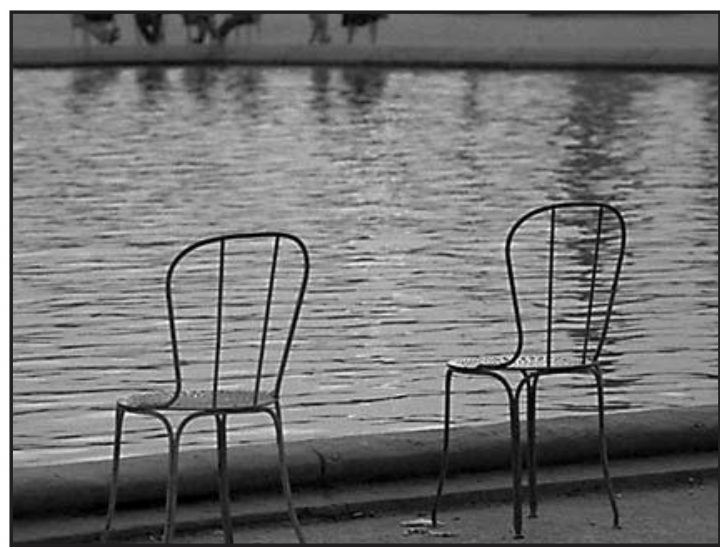

HISTORIOGRAPHY OF NURSING IN SPAIN: THE STORY OF A SMALL-GREAT FAMILY

\section{SUMMARY}

7 he main target focuses in the way research of Nursing History allows us today to mention such a simple heading 'Historiography', if we consider the extreme rigour general History demands on analyzing different trends along centuries to deal with knowledge and command of methods and auxiliary sciences. But actions have been taken and we do have confidence that the new Study Programmes and new research possibilities locate History in its real place.

We do start from the query What does History mean?. As much the query as historical truth have been following a line along centuries. From Herodote, Tucidide, Tito Livio we are able to follow different critical reviews and tendencies from the experts. It is worth while as an example remembering Voltaire's evaluation in his study on History in his Encyclopaedia: 'Have you nothing but to say a barbarian followed another barbarian in the Oxus and Jaxartes banks, what daes it matter?'. Simple positive research, objective compilation and contrasted facts.
Some other data situates us facing aspects of History: Miguel de Unamuno's text: Undoubtedly a History book could have no mistaken data or no wrong references but it could be a deep lie all together, or on the contrary, it could be a faithful exposé of reality and be an absolute inaccuracy, although I am not defending such an statement (Miguel de Unamuno). Those texts make us think.

Key words: History, Nursing. Historiography

HISTORIOGRAFIA DA ENFERMAGEM NA ESPANHA: A HISTÓRIA DE UMA PEQUENA-GRANDE FAMÍLIA

\section{RESUMO}

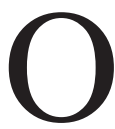
objetivo é saber como a investigação da História da Enfermagem nos possibilita hoje falar de Historiografia da mesma. Esta afirmação pode chocar com o rigor que se tem posto à História em geral, ao analisar as múltiplas correntes da mesma que através dos séculos têm surgido, bem como a sua relação com o conhecimento e domínio que os métodos e ciências auxiliares reclamam hoje, mas os passos estão sendo dados e confiamos que os novos planos de estudo, e a possibilidade de investigar, situem a História em seu lugar.

Parto da pergunta acerca da História: o que é a História? A pergunta pela verdade histórica tem sido uma constante através dos séculos: Desde Heródoto, Tucídides, Tito Lívio, podemos observar as críticas e tendências dos críticos sobre a mesma. Vale a pena relembrar - a título de exemplo - a valorização de Voltaire, em seu artigo sobre a "História" na Enciclopédia. "Se nada tivéreis a dizer-nos salvo que um bárbaro sucedeu a outro nas ribeiras do Oxus e de Jaxartes? O que mais dá? Simples investigação positiva: compilação objetiva e documentada de fatos. 
Outro dado que nos localiza diante de alguns aspectos da História é este texto de Miguel de Unamuno: "É inquestionável que um livro de história pode não conter nem um dato falso nem uma referência equivocada, e ser, entretanto, uma mentira em seu conjunto, e que, pelo contrário, pode dar-nos um fiel reflexo da verdade e estar repleto de inverdades. O qual não é defender estar" (Miguel de Unamuno). Estes textos nos dão que pensar.

Palavras-chave: História, Enfermagem, Historiografia.

\section{RESUMEN}

$\mathrm{E}$ 1 objetivo se centra en saber cómo la investigación de la Historia de la Enfermería nos permite hoy hablar de Historiografía de la misma. Esta afirmación puede chocar con el rigor que se ha pedido a la Historia en general, al analizar las múltiples corrientes de la misma que a través de los siglos han surgido y en su relación al conocimiento y dominio que los métodos y ciencias auxiliares reclaman hoy, pero los pasos están dados y confiamos que los nuevos planes de estudio, y la posibilidad de investigar sitúen la Historia en su lugar.

Parto de la pregunta por la Historia ¿qué es la Historia?. La pregunta por la verdad histórica ha sido una constante a través de los siglos: Desde Heródoto, Tucídides, Tito Livio podemos observar las críticas y tendencias de los críticos acerca de la misma. Vale la pena recordar - a modo de ejemplo - la valoración de Voltaire en su artículo sobre la "Historia" en la Enciclopedia. "Si nada tenéis que decirnos salvo que un bárbaro sucedió a otro en las riberas del Oxus y de Jaxartes, ¿Qué más da?". Simple investigación positiva: compilación objetiva y documentada de hechos.

Otro dato que nos sitúa ante algunos aspectos de la Historia es este texto de Miguel de Unamuno: "Es indudable que un libro de historia puede no contener ni un dato falso ni una referencia equivocada, y ser, sin embargo, una mentira en su conjunto, y que, por el contrario, puede darnos un fiel reflejo de la verdad y estar plagado de inexactitudes. Lo cual no es defender estas" (Miguel de Unamuno). Estos textos nos dan que pensar.
Palabras clave: Historia, Enfermería, Historiografía

\section{LA HISTORIA, SU FUNCIÓN Y SU MÉTODO}

Adentrándose en la Historia, vemos cómo, su función, hasta muy recientemente no ha sido reconstruir del modo más exacto y fidedigno el pasado. Esta concepción es muy moderna, ya que tiene escasamente dos siglos de vigencia. Esta ha llegado por la Escuela Alemana y principalmente por Dilthey (1986). La función del cronista y del historiador tradicional ha consistido en cantar las obras y hazañas de los personajes importantes. De ahí que Historia y leyenda se identificaban. ¿Qué son los poemas homéricos, Historias, leyendas?Ambas cosas.

La Historia del arte como otras historias y entre ellas la historia de la medicina hasta hace dos siglos ha sido esto. Historia y leyenda y como prototipo ahí tenemos el mito de Hipócrates. Algo similar aunque en menor medida hemos hecho nosotras con la Enfermería creando el Mito de Florence Nightingale. Hemos narrado los hitos de la Enfermería, pero como no han tenido tanta trascendencia hemos hecho un esfuerzo por investigar esa pequeña historia de la gran historia, con la que, aun siendo verdadera, no estamos satisfechas; pero sabemos que tendrá su lugar y su tiempo.

De otro lado, desde hace unas décadas se ha puesto de moda en la literatura sobre profesionalismo y ética de las profesiones, hablar de lo que ha dado en llamarse "moralidad interna". Todos los roles sociales tienen una finalidad interna, que viene definida por su propia actividad. Además, como se trata de moralidad, lo que define no es tanto el "ser" de la profesión, sino el modo cómo deberían comportarse los profesionales. Esta adecuación siempre es difícil y nunca acaban de identificarse y la historia se ha escrito desde el "deber ser".

Las cosas comenzaron a cambiar en la época de la Ilustración, en el siglo XVIII. Es entonces cuando se descubre la historia como tal y se intenta convertirla en disciplina estricta y rigurosa. Su objetivo va a ser la fijación de los hechos pasados, deslindando los hechos históricos de todas aquellas otras cosas que no son hechos estrictos. Esto exigi- 
ría poner a punto un método riguroso de identificación y análisis de los hechos históricos. Este método adquirirá forma canónica en el siglo XIX, el llamado "método histórico-crítico", si bien ya está perfectamente constituido por autores como Droysen quien en su HistoriK aparecen ya sus tres pasos preceptivos o canónicos:

- La Heurística, ciencia de las fuentes. Búsqueda, catalogación y manejo de las mismas.

- La crítica. Lo que estas dicen hay que someterlo a un proceso de depuración.

- Hermenéutica, la interpretación.

Por tanto, la función del Historiador es reconstruir el pasado, contar las cosas como ellas fueron. Pero el historiador sabe que el reconstruir el pasado es imposible. Nunca seremos capaces de hacerlo de modo acabado, ni reconstruir el pasado tal como él fue porque la razón humana no sólo es lógica, sino también histórica y que si bien la historia no es del todo racional, la razón si es formalmente histórica. De ahí que la Historia es un modo de conocimiento. El ocultar o eliminar la dimensión histórica es eliminar una de las principales herramientas que tiene la razón humana para entender y comprender las cosas. Exige rigor y precisión. La Historia permite que conozcamos mejor nuestro pasado y nos conozcamos algo mejor a nosotros mismos. Adquirir conciencia de los orígenes, asumir la herencia de los antepasados y procurar, con la propia vida mejorarla y recrearla.

Y son los grandes pensadores del XIX quienes contribuyen a crear otra forma de interpretar la Historia y sobre todo a crear conciencia sobre la Historia, entre ellos H. Gadamer (2003) con su obra Verdad y Método ha contribuido a ello.

Lo esencial no sólo se encuentra en el retener o en el olvidar, sino en la tensión interna, esa plástica fuerza que nos permite situarnos ante el pasado como algo que nos es propio, nos constituye y nos lanza hacia el futuro.

La hermenéutica los piensa conjuntamente. El problema de la conciencia histórica no es sólo el problema de la verdad en el seno del devenir histórico. Se trata, básicamente, de responder a la pregunta de cómo y en virtud de qué se conforma la voluntad humana en una voluntad memorante, es decir, en una voluntad de responsabilidad histórica.

\section{LA INCORPORACIÓN DE LA HISTORIA DE LA ENFERMERÍA A LA HISTORIO- GRAFÍA}

Partimos de la premisa de que no existe grupo humano amnésico, esta circunstancia le privaría de su existencia. La historia así se convierte en "la memoria", entendida como el modo discursivo escrito y con intenciones explicativas a través del cual se pretende dar cohesión lógica a lo acaecido, con aspiraciones de certeza. Se le otorga así al pasado una dimensión científico-explicativa, que evoluciona según los diferentes paradigmas en que se inscribe, para relatar los acontecimientos del pasado a modo de testimonio.

Será pues la Historiografía, -la historia de la Historia-, la que nos conduzca en este empeño por aproximarnos a la disciplina enfermera y contribuir a dilucidar el camino de intención propedéutica necesario para su construcción científica, a través de conversaciones con los paradigmas científico-explicativos de la historia.

Esta exigencia histórica, entendida como la necesidad de preservar del olvido las tradiciones del pasado, nos obliga a recapacitar acerca de las últimas causas que dan origen a la historiografía en Grecia. Porque es el propio término Historia, que significa "saber" por haber visto u oído algo, el que nos conduce a la precisa acepción del término griego que significa, asimismo, información, averiguación y en definitiva resultado de una investigación.

Sabemos que la Historia de la Enfermería es la historia de los cuidados, todo un mundo al que no se ha prestado atención por parte de los historiadores hasta muy recientemente. La Historia de la medicina se fijó tan sólo en la dimensión de la "curación" del acto sanitario, desatendiendo casi completamente la de "cuidado", cuando la función esencial de todo acto sanitario es la de "cuidado".

Ha sido en el último tercio el siglo XX cuando la enfermería toma conciencia que tiene una larga historia y que es necesario escribirla porque el cuidado ha sido fundamental para la sociedad. Si bien, la Historia como saber diferenciado tuvo que construir herramientas con las que construir el pasado, la Enfermería ha comenzado a utilizarlas y con ello ha nacido la pasión de historiar un oficio que tiene un legado bi-milenario.

Pero ha sido en estos treinta últimos años cuando se ha empezado a desarrollar esta realidad, 


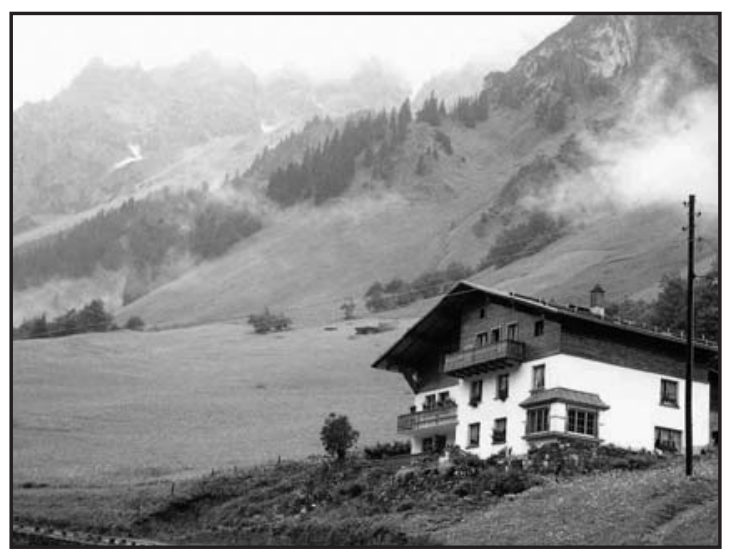

haciendo que El anclaje de este armazón esté en la corriente historiográfica que presenta la historia no como relato, sino como problema, no centrada tanto en el texto como en la amplitud de las fuentes, en las sencillas pero sorprendentes actividades cotidianas del hombre en sociedad, en el cuidado. Este es nuestro compromiso y nuestra meta.

De ahí que, hablar de Historiografía en enfermería coincida con la toma de conciencia de la Historia de la Enfermería, en la segunda mitad del siglo XX y con la reclamación del estatus profesional. Las publicaciones de Historia de la Enfermería de Jamieson, $M^{a}$ Teresa Molina y Frank Elizondo coinciden con este momento y suponen un paso trascendental. Coincide en España con el desarrollo de los Planes de Estudio de los Ayudantes Técnicos Sanitarios y la creación de las Escuelas de Enfermería. En el segundo curso aparece la Historia de la Enfermería como asignatura a impartir. No sé si en todas la Escuelas se llevó a cabo y por quién; posiblemente médicos y ahí se justifica el fascículo del Dr. José Álvarez Sierra.

Pasados unos años y coincidiendo con el reconocimiento de la Enfermería como carrera universitaria, son los Hermanos de San Juan de Dios Francisco Ventosa y Basilio Eseverri Chaverri y Carmen Domínguez Alcón, quienes marcan los primeros pasos como historiadores de la enfermería en España. Esto, junto con la introducción de la Historia en los planes de Estudios será otro paso importante en el despertar de la conciencia de la Historia de la Enfermería y me atrevería a decir la conciencia de contar la Historia de la Enfermería.

Sin embargo, la conciencia de que hay que investigar la Historia para escribir la historia y enseñarla surge unos años más tarde. Con los planes de estudios universitarios se inicia su enseñanza, pero como una parte de los Fundamentos de la Enfermería. Poco a poco se desarrolla un programa para ser explicada de forma independiente como asignatura cuatrimestral en algunas Escuelas.

Es en estos años, hacia 1985, cuando se empieza a gestar en la Escuela Universitaria de Enfermería de la Complutense un premio a la Investigación en Historia de la Enfermería, primero Nacional y después Internacional. Este hecho coincide con la creación de la asignatura de Historia de la Enfermería, obligatoria en el centro y con la creación del Seminario Permanente para la Investigación de la Historia de la Enfermería en 1989.

El paso definitivo se daba en la Jornada sobre el Método de Investigación Histórica. Se abría un nuevo horizonte a la Historia y a la Investigación Histórica con la presentación del Proyecto al que se adherían profesoras de las diversas Escuelas de Comunidades Autónomas y el que seguimos paso a paso con Jornadas y Congresos. Surge así un interés por la investigación de la Historia porque los textos con que contábamos en esos momentos no respondían a las exigencias de unos estudios universitarios y con ello surgía una promoción de Historiadores de la Enfermería.

Estos pasos iniciales y sencillos, han consolidado este proyecto que hoy celebra, tras sus modestos inicios con cursos y Jornadas, su $\mathrm{X}$ Congreso Nacional y V Internacional en este 2008. Sus publicaciones son testigos de ello. Se han abordado las etapas secuenciales de la Historia, las etapas del desarrollo y contenido de la profesión y conectado con acontecimientos históricos importantes.

Sin embargo, a pesar de sus modestos logros, hoy se hace necesaria una revisión pues los criterios, han combinado rigor, flexibilidad y motivación. Se ha puesto interés y pasión, pero en estos momentos la medida será la calidad y la excelencia. Entre sus repercusiones hacemos referencia a tres aspectos: Primero: Se ha despertado la conciencia de la Historia. Y esto ha tenido repercusión en la enseñanza de la misma, estableciendo programas de enseñanza obligatoria, de libre configuración, etc. Segundo: Se han creado plazas para profesorado especializado (titularidades y Cátedras) y Tercero: En el interés por la investigación. 
No podemos hacer en este artículo un elenco acerca de todas las publicaciones y sus autores, pero ha habido un gran interés por:

- Publicación de textos de Historia de la Enfermería para Alumnos.

- Revistas sobre Historia del Cuidado e Historia de la Enfermería.

- Revistas electrónicas

- Bases de Datos.

- Todo esto refleja el interés por nuestra Historia.

Con el trabajo realizado por todos creemos haber contribuido al desarrollo de la Historiografía de la Enfermería en España, sabiendo que dejamos a los futuros historiadores e investigadores una puesta abierta y muchos archivos por desempolvar. De ahí que a la Historia de la Enfermería, olvidada anteriormente, le espera un futuro prometedor, aunque no exento de dificultades que sino se preveen y se ponen los remedios a tiempo, puedan resultar insalvables

\section{BIBLIOGRAFÍA}

- AUBERT, N. y GAULEJAC, V. (1993) El coste de la excelencia, Barcelona: Paidós.

- CARBONELL, Charles-Olivier (1986) La Historiografía. Fondo de Cultura Económica, México.

- CASSIRER, E. (1990) La hermenéutica del lenguaje. Bilbao: Universidad de Deusto.

- CASSIRER, E. (1997) Antropología filosófica. Introducción a una filosofía de la cultura, México: Fondo de cultura económica.

- COMELLES, JM. (1992) Cuidar y curar. Bases para una historia antropológica de la enfermería hospitalaria. Rol (172):35-41.

- CONSEJO INTERNACIONAL DE ENFERMERAS (1961) Principios Básicos de los cuidados de Enfermería. Washington: Organización Panamericana de la Salud, OMS., (Publicación científica, $n^{\circ}$ 57).

- DILTHEY, W.(1986) Introducción a las ciencias del espíritu: Ensayo de una fundamentación del estudio de la sociedad y de la historia, Madrid: Alianza.

- DOMINGUEZ-ALCON, C. (1986) Los cuidados y la profesión de Enfermería en España. Madrid. Pirámide.

- DONAHUE, M. P. (1989) Historia de la Enfermería, Barcelona: Doyma.

- ELLIOT, P. (1975) Sociología de las profesiones. Madrid: Tecnos.

- GADAMER, H-G. (2003) Verdad y método, Salamanca: Sígueme.

- GADAMER, H-G. (1993) El problema de la conciencia histórica, Madrid: Tecnos.

- GARCIA GUAL (1992) Introducción a la mitología griega, Madrid: Alianza.
- GONZÁLEZ ANLEO, J. (1994) Las profesiones en la sociedad corporativa, en 15. FERNÁNDEZ, JL. y HORTAL, A. (coms) "Ética de las profesiones", Madrid: Univ. Comillas.

- GRACIA GUILLÉN, D. (1996) Prólogo. En "Historia de la Enfermería en España: desde la antigüedad hasta nuestros días". Madrid: Síntesis.

- GRACIA GUILLÉN, D. (2004) Como arqueros al blanco. Madrid: Triacastela.,(La medicina en el cambio de siglo).

- HENDERSON, V. (1988) Enfermería: Teoría y práctica. (3 vol). La Prensa Médica Mexicana. México.

- HENDERSON, V. (1994) La naturaleza de la Enfermería: Reflexiones 25 años después. Interamericana-McGraw-Hill.

- HERNÁNDEZ CONESA, J. M., MORAL DE CALATRAVA, P., ESTEBAN ALBERT, M. (2003) Fundamentos de la enfermería. Teoría y método, Madrid: $2^{\mathrm{a}}$ ed., McGraw-Hill Interamericana.

- HERNÁNDEZ MARTÍN, F. (Coord.) (1996) Historia de la Enfermería en España (desde la antigüedad hasta nuestros días), Madrid: Síntesis.

- HERNÁNDEZ MARTÍN, F.; GALLEGO LASTRA, R. del; ALCARAZ GONZÁLEZ, S.; GONZÁLEZ RUIZ, JM. (1997)

"La Enfermería en la Historia: Un análisis desde la perspectiva profesional", Cultura de los Cuidados, 2: 21-35.

- HERNÁNDEZ MARTÍN, F.; GALLEGO LASTRA, R. (1999) V. Henderson: Autora clave para la enfermería actual. Híades, 5-6: 11-24. Alcalá de Guadaira.

- KÉROUAC, S., PEPIN, J., DUCHARME, F., DUQUETTE, A., MAJOR, F. (2005) El pensamiento enfermero, $3^{\mathrm{a}}$ reimp., Barcelona: Masson.

- LAIN ENTRALGO, P. (1972) Historia Universal de la Medicina. Barcelona: Salvat.

- MEDINA, JL. (1998) La pedagogía del cuidado: saberes y prácticas en la formación universitaria en enfermería, Barcelona: Alertes.

- MEDINA MOYA, JL., SANDIN ESTEBAN, MP.(1994) "Fundamentación epistemológica de la teoría del cuidado", Enfermería Clínica, 4 (5): 221-231.

- MEDINA MOYA, JL., SANDIN ESTEBAN, MP. (1995) Epistemología y Enfermería: Paradigmas de la investigación en Enfermería. Enfermería Clínica, 5(1): 32-44.

- PARSONS, T. (1976) El sistema social, 2a ed. Madrid: Revista de occidente.

- PETERS, TJ. , WATERMAN, RH. (1990) En busca de la excelencia, Barcelona: Folio.

- POLIT-HUNGLER. (1995) Investigación científica en Ciencias de la Salud. 5a ed, México: Mc-Graw-HillInteramericana.

- RICOEUR, P. (1976) Sentido y existencia: Homenaje a Paul Ricoeur. Gray Brent, Madisson. Estella: Ed. Verbo Divino.

- SÁNCHEZ GONZÁLEZ, MA. (1998) Historia, teoría y método de la medicina. Introducción al pensamiento médico, Barcelona: Masson.

- SCHIPPERGES, H. (1987) En el jardín de la salud. Barcelona: Laila.

- SMITH, J. P. (1996) Virginia Henderson. Los Primeros noventa años. Barcelona: Masson.

- TORRALBA, F. (1999) Antropología del cuidado, Madrid: MAFRE.

- MACINTYRE, A.(1987) Tras la virtud, Crítica, Barcelona. 